\title{
Medical Image of the Week: Chronic Bilateral Fibrocavitary Pulmonary Coccidioidomycosis
}

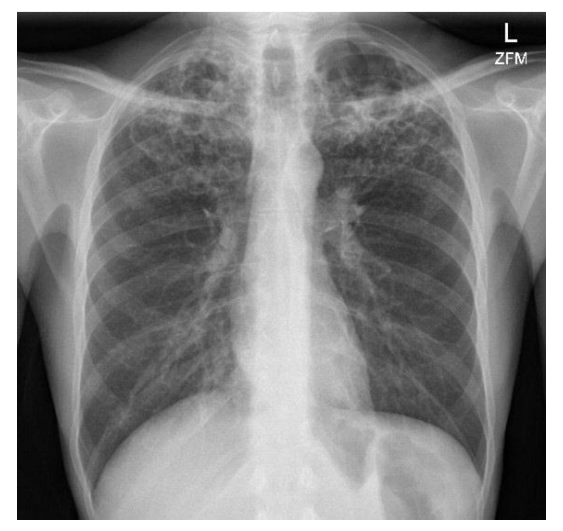

Figure 1. Chest x-ray showing bilateral upper lobe cavitation.

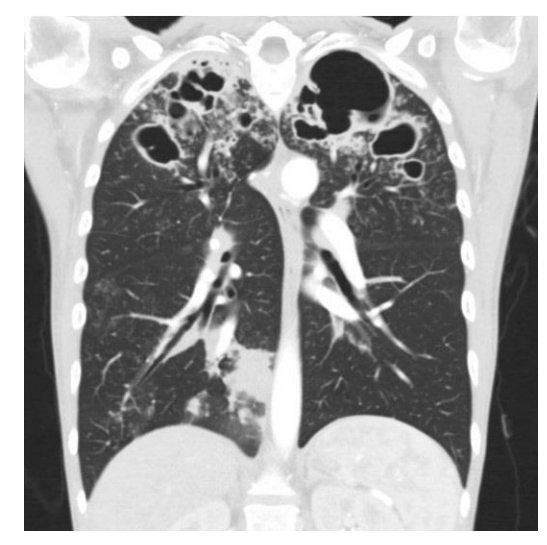

Figure 2. Thoracic computed tomography showed bilateral upper lobe, multiple thickwalled cavities with associated tree in bud opacities and consolidation in the right lower lobe.

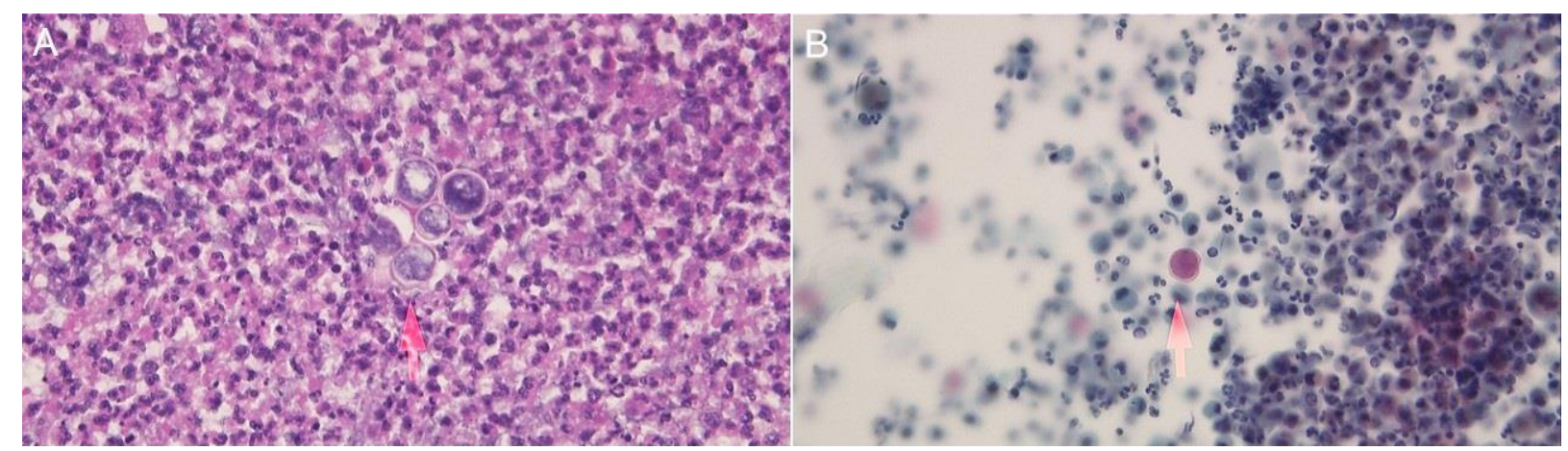

Figure 3. Cytology of bronchoalveolar lavage fluid showing coccidioidomycosis spherules (arrows). 
A 47-year-old Ethiopian immunocompetent male with no past medical history presented with cough and blood tinged sputum for 1 month with no fever, night sweats, or weight loss. Chest X-ray showed bilateral upper lobe cavitary lesions (Figure 1). Computed tomography of the chest showed bilateral upper lobe, multiple thick-walled cavities with associated tree in bud opacities and consolidation in the right lower lobe (Figure 2). TB was ruled out and a bronchoalveolar lavage (BAL) was performed. Cytology on the BAL showed the presence of Coccidioides immitis spherules (Figure 3). Serum coccidioidomycosis by complement fixation 1:64 with positive IgG by immunodiffusion; serum antigen by EIA 0.30; and urine antigen was negative. The patient was started on fluconazole and was discharged with pulmonology follow up. Bilateral cavitary lesions are rare and they can mimic the reactivation of $M$. tuberculosis, reinforcing the importance of including coccidioidomycosis in the differential diagnosis of bilateral cavitary lung lesions for patients in endemic areas.

Tarreq Noori MD*, Mohammed Al-Charakh MD*, and Andres Borja Alvarez MD** Departments of Internal Medicine* and Pulmonology** Maricopa Integrated Health System Phoenix, AZ USA

\section{Reference}

1. Jude CM, Nayak NB, Patel MK, Deshmukh M, Batra P. Pulmonary coccidioidomycosis: pictorial review of chest radiographic and CT findings. Radiographics. 2014 Jul-Aug;34(4):912-25. [CrossRef] [PubMed] 